P.C. HU

KODAI MATH. J.

13 (1990), 349-362

\title{
A MODIFIED DEFECT RELATION FOR HOLOMORPHIC CURVES
}

\author{
BY PEICHU HU
}

\section{Introduction and main results.}

By a holomorphic curve, we mean a holomorphic mapping

$$
x: V \longrightarrow \boldsymbol{P}_{n},
$$

where $V$ is an open Riemann surface and $\boldsymbol{P}_{n}$ is the $n$-dimensional complex projective space. In 1927, R. Nevanlinna [3] created a new theory concerning the distribution of values of a holomorphic curve $f: \boldsymbol{C} \rightarrow \boldsymbol{P}_{1}$. Nevanlinna's main result is that $f$ assumes almost all values in $\boldsymbol{P}_{1}$ "equally often", and those values that $f$ fails to assume often enough have total "defect" at most 2 . H. Cartan [2] generalized this "defect relation" to holomorphic curves $x: C \rightarrow \boldsymbol{P}_{n}$ counting how often $x$ takes values in hyperplanes. L. Ahlfors [1] later extended Cartan's result to holomorphic curves $x: V \rightarrow \boldsymbol{P}_{n}$, which he cast in a geometric form. $\mathrm{H}$. Wu [5] reorganized Ahlfors' theory in a modern fashion. We freely use the symboles, notations and terminologies from $\mathrm{H}$. Wu [5] except for special declaration.

The purpose of this paper is to modify the Second Main Theorem for holomorphic curves, and furthermore, simplify the defect relation. Let $\tau$ be a harmonic exhaustion on $V$ and $\sigma=\tau+\sqrt{-1} \rho$ be the special coordinate function. By a theorem of Gunning and Narasimhan [5, p. 102], there is a holomorphic function $\gamma$ on $V$ whose differential vanishes nowhere. Thus in every sufflciently small open subset of $V$, the restriction of $\gamma$ to it is a coordinate function. Define

$$
\begin{aligned}
& H(r)=\left.\frac{1}{2 \pi} \int_{\partial V[t]} \log \left|\frac{d \sigma}{d \gamma}\right| * d \tau\right|_{r_{0}} ^{r}, \\
& T_{k}^{0}(r)=T_{k}(r)+N_{k}(r),
\end{aligned}
$$

Partially supported by the National Science Foundation.

Key words and phrases. Holomorphic curves, Defect relation.

1980 Math. Subject Classification. 32A22, 30D35.

Received October 30, 1989; revised February 23, 1990. 


$$
\varepsilon_{k}^{q}= \begin{cases}1, & \text { if } 0 \leqq q \leqq k \\ \frac{(n-q)(k+1)}{(n-k)(q+1)}, & \text { if } k \leqq q \leqq n-1,\end{cases}
$$

Then we obtain

THEOREM 1. Let $x: V \rightarrow \boldsymbol{P}_{n}$ be a nondegenerate holomorphic curve and $V$ admits a harmonic exhaustion, then for $k=0, \cdots, n-1$

$$
E(r)+H(r)+S_{k}(r)=N_{k-1}(r)-2 N_{k}(r)+N_{k+1}(r)
$$

$$
T_{k-1}^{0}-2 T_{k}^{0}+T_{k+1}^{0}=H+\mu(T)
$$

and for $k=1, \cdots, n$

$$
N_{k}(r)=(k+1) N_{0}(r)+\frac{k(k+1)}{2}(E(r)+H(r))+\sum_{j=0}^{k-1}(k-j) S_{j}(r) .
$$

THEOREM 2. Let $x: V \rightarrow \boldsymbol{P}_{n}$ be a nondegenerate holomorphic curve and $V$ admits a harmonic exhaustion. Let $\left\{A^{q}\right\}$ be a finite system of q-dimensional projective subspaces of $\boldsymbol{P}_{\boldsymbol{n}}$ in general position. Then the generalized compensating terms $m_{k}\left(A^{q}\right)=m_{k}\left(r, A^{q}\right)$ satisfy the following inequality

$$
\sum_{A^{q}} m_{k}\left(A^{q}\right)=\varepsilon_{k}^{q}\left(\begin{array}{l}
n \\
q
\end{array}\right)\left(\frac{n+1}{k+1} T_{k}^{0}-N_{n}+\frac{1}{2}(n+1)(n-k) H\right)+\mu\left(T^{2}\right) .
$$

We also have the equality

$$
\begin{aligned}
& \frac{n+1}{k+1} T_{k}^{0}(r)-N_{n}(r)+\frac{1}{2}(n+1)(n-k) H(r) \\
& \quad=\frac{n+1}{k+1} T_{k}(r)-Q_{k}(r)-\frac{1}{2}(n+1)(n-k) E(r) .
\end{aligned}
$$

where

$$
Q_{k}(r)=\frac{n-k}{k+1} \sum_{j=0}^{k-1}(j+1) S_{j}(r)+\sum_{j=k}^{n-1}(n-j) S_{j}(r)
$$

Remark. If $\tilde{x}=\left(x_{0}, \cdots, x_{n}\right): V \rightarrow C^{n+1}$ is a reduced representation of $x$, then

$$
N_{n}(r)=\int_{r_{0}}^{r} n(t, W=0) d t
$$

where $W=W\left(x_{0}, \cdots, x_{n}\right)$ is the Wronskian determinant of $x_{j}(j=0, \cdots, n)$ and $n(t, W=0)=$ sum of the orders of zeroes of $W$ in $V[t]$.

Thus if $V=C$ and $k=0,(4)$ is just the Cartan's Second Main Theorem [2], [4].

I learned about differential geometry and complex analysis from $\mathrm{H}$. Wu and

Y.T. Siu, whom I wish to thank for sharing their insights with me. 


\section{Proof of Theorem 1 .}

Given a holomorphic curve $x: V \rightarrow \boldsymbol{P}_{n}$, with a reduced representation $\tilde{x}=$ $\left(x_{0}, \cdots, x_{n}\right): V \rightarrow C^{n+1}$. According to $\mathrm{H}$. Wu [5] the quantity $X_{z}^{k}$ is defined as follows: fix a coordinate neighborhood $U$ in $V$ and a coordinate function $z$ on $U$,

$$
X_{z}^{k}=\tilde{x} \wedge \tilde{x}^{(1)} \wedge \cdots \wedge \tilde{x}^{(k)}, \quad k=0, \cdots, n,
$$

where $X_{z}^{0}=\tilde{x}^{(0)}=\tilde{x}$ and

$$
\tilde{x}^{(i)}=\left(\frac{d^{2} x_{0}}{d z^{2}}, \cdots, \frac{d^{2} x_{n}}{d z^{2}}\right),
$$

Then the following results are well-known [5]

$$
\begin{gathered}
X_{\gamma}^{k}=\left(\frac{d \sigma}{d \gamma}\right)^{k(k+1) / 2} X_{\sigma}^{k} \quad[5, \text { p. } 69] \\
T_{k}(r)=\left.\frac{1}{2 \pi} \int_{\left.\partial V_{i} t\right]} \log \left|X_{\gamma}^{k}\right| * d \tau\right|_{r_{0}} ^{r}-N_{k}(r) \quad[5, \text { p. } 104] \\
E(r)+S_{k}(r)+T_{k-1}(r)-2 T_{k}(r)+T_{k+1}(r) \\
=\left.\frac{1}{2 \pi} \int_{\left.\partial V_{[} t\right]} \log \frac{\left|X_{\sigma}^{k-1}\right|\left|X_{\sigma}^{k+1}\right|}{\left|X_{\sigma}^{k}\right|^{2}} * d \tau\right|_{r_{0}} ^{r} \quad[5, \text { p. 130] } \\
E+S_{k}+T_{k-1}-2 T_{k}+T_{k+1}=\mu(T) . \quad[5, \text { p. 132] }
\end{gathered}
$$

where (7) holds in $V-V[r(\tau)]-\{$ critical points of $\tau\}$. Since $r_{0} \geqq r(\tau)$ and an integration always ignores finite point sets (the critical points of $\tau$ are all isolated), by (7) and (8), we have.

$$
\left.\frac{1}{2 \pi} \int_{\left.\partial V_{[} t\right]} \log \left|X_{\sigma}^{k}\right| * d \tau\right|_{r_{0}} ^{r}=T_{k}(r)+N_{k}(r)-\frac{1}{2} k(k+1) H(r) .
$$

Consequently, (9) and (11) imply (1).

Note that $N_{-1}(r)=0$. So upon (1) summing over $k$ from 0 to $j-1$, we have:

$$
j(E(r)+H(r))+\sum_{\imath=0}^{j-1} S_{i}(r)=N_{j}(r)-N_{\jmath-1}(r)-N_{0}(r) .
$$

Upon summing over $j$ from 1 to $k$, we finally have (3). (1) and (10) imply (2). q.e.d.

\section{Preliminary lemmas.}

To prove Theorem 2 we need some lemmas.

LEMMA $1[5$, p. 131$]$. (i) If $\phi_{1} \leqq \phi$ off a compact set and $\varphi \leqq \varphi_{1}$ off a compact 
set, then $\phi=\mu(\varphi)$ implies $\phi_{1}=\mu\left(\varphi_{1}\right)$.

(ii) If $\phi=\mu(\varphi)$, then $\phi+O(1)=\mu(\varphi)$.

(iii) If $C$ is a positive constant and $\phi=\mu(\varphi)$, then

$$
C \psi=\mu(\varphi) \text {. }
$$

(iv) If $\phi=\mu(\varphi)$ and $\phi_{1}$ is positive off a compact set, then

$$
\phi-\phi_{1}=\mu(\varphi) \text {. }
$$

(v) Suppose $\phi=\mu(\varphi)$ and $\phi_{1}=\mu(\varphi)$. then $\phi+\phi_{1}=\mu(\varphi)$. only if

Remark. We say $\phi=\mu(\varphi)$ for two continuous functions $\varphi$ and $\phi$ if and

$$
\int_{r_{0}}^{r} d s \int_{r_{0}}^{s} \exp \{K \psi(t)\} d t<C \varphi(r)+C^{\prime} \quad[5, \text { p. 131] }
$$

for some positive constants $K, C$ and $C^{\prime}$.

LEMMA 2. For $k=0, \cdots, n-2$,

$$
(k+1) T_{k+1}^{0}=(k+2) T_{k}^{0}+\frac{1}{2}(k+1)(k+2) H+\mu(T)
$$

and for $k=1, \cdots, n-1$,

$$
(n-k) T_{k-1}^{0}=(n-k+1) T_{k}^{0}+\frac{1}{2}(n-k)(n-k+1) H-N_{n}+\mu(T) .
$$

Proof. By (2) and Lemma 1 (iii), we have

$$
(k+1)\left(T_{k-1}^{0}-2 T_{k}^{0}+T_{k+1}^{0}-H\right)=\mu(T)
$$

and

$$
(n-k)\left(T_{k-1}^{0}-2 T_{k}^{0}+T_{k+1}^{0}-H\right)=\mu(T) .
$$

Upon (15) and (16) summing over $k$ from 0 to $k$ and $k$ to $n-1$ respectively, and using Lemma 1 (v), we get (13) and (14). q.e.d.

COROLLARY 1. If $j \geqq k$, then

$$
(k+1) T_{j}^{0}=(j+1) T_{k}^{0}+\frac{1}{2}(j-k)(k+1)(j+1) H+\mu(T) .
$$

If $j \leqq k$, then

$$
(n-k) T_{j}^{0}=(n-j) T_{k}^{0}+\frac{1}{2}(k-j)(n-k)(n-j) H-(k-j) N_{n}+\mu(T) .
$$

Proof. Straightforward induction from the lemma. 
COROLLARY 2. If

$$
H_{k}=\lim _{r \rightarrow \infty} \sup \frac{H(r)}{T_{k}^{0}(r)}<+\infty,
$$

where we assume that $V$ has an infinite harmonic exhaustion and that $x$ is nondegenerate, then there exists a positive constant $c$ such that

$$
\| T_{k}^{0}(r) \leqq T^{0}(r) \leqq c T_{k}^{0}(r),
$$

where $T^{0}(r)=\max \left\{T_{0}^{0}(r), \cdots, T_{n-1}^{0}(r)\right\}$, and the sign "\|" in front of an inequality means that the inequality is only valid in $[0, \infty)-I$ with $\int_{I} d \log t<\infty$.

Proof. We know that $\phi=\mu(\varphi)$ implies

$$
\| \phi(r)<\lambda \log \left(C \varphi(r)+C^{\prime}\right)
$$

for a constant $\lambda>1([5],(4.62))$. Hence (17) and (18) imply

$$
\begin{aligned}
\|(k+1) T_{j}^{0}(r) & <(j+1) T_{k}^{0}(r)+\frac{1}{2}(j-k)(k+1)(j+1) H(r) \\
& +\lambda \log \left(C T(r)+C^{\prime}\right) \quad \text { if } j \geqq k
\end{aligned}
$$

and

$$
\begin{gathered}
\|(n-k) T_{j}^{0}(r)<(n-j) T_{k}^{0}(r)+\frac{1}{2}(k-j)(n-k)(n-j) H(r) \\
+\lambda \log \left(C T(r)+C^{\prime}\right) \quad \text { if } j \leqq k .
\end{gathered}
$$

Obviously, they together imply that for some positive constants $c_{1}$ and $c_{2}$.

$$
\| T^{0}(r)<c_{1} T_{k}^{0}(r)+c_{2} \log \left(C T^{0}(r)+C^{\prime}\right) \cdot
$$

Because $T_{k}(r) \rightarrow \infty$ as $r \rightarrow \infty$, so $T^{0}(r) \rightarrow \infty$ as $r \rightarrow \infty$. Thus for sufficiently large $r$,

$$
c_{2} \log \left(C T^{0}(r)+C^{\prime}\right)>\frac{1}{2} T^{0}(r) .
$$

Combining with the above inequalities, we obtain (19).

q.e.d.

REMARK. If $x$ is nondegenerate and $V$ has an infinite harmonic exhaustion and

$$
\lambda_{k}=\lim _{r \rightarrow \infty} \sup \frac{-E(r)}{T_{k}(r)}<+\infty .
$$

we also have

$$
\| T_{k}(r) \leqq T(r) \leqq c T_{k}(r) . \quad[5, \text { p. } 140]
$$


LEMMA 3. If $y_{j}$ are indeterminates over the ring $\boldsymbol{Z}$ and if $y_{j}=0$ for $j>n$, then we have the algebraic identity

$$
\begin{aligned}
& D_{q}(k, l ; y) \equiv-\sum_{j=k}^{n-1} \sum_{\imath=0}^{l} P_{q}(j-i, l-i)\left(y_{\jmath-\imath-1}-2 y_{\jmath-i}+y_{\jmath-\imath+1}\right) \\
& =P_{q}(k+1, l+1) y_{k}-\left(P_{q}(n+1, l+1)-\left(\begin{array}{c}
n \\
l+1
\end{array}\right)\left(\begin{array}{c}
-1 \\
q-l
\end{array}\right)\right) y_{n} \\
& \quad-\sum_{\imath=k-l-1}^{k}\left(\begin{array}{c}
i \\
l-k+i+1
\end{array}\right)\left(\begin{array}{c}
n-i-1 \\
q+k-l-i
\end{array}\right) y_{i}-\sum_{\imath=k+1}^{n}\left(\begin{array}{c}
i \\
l
\end{array}\right)\left(\begin{array}{c}
n-i-1 \\
q-l-1
\end{array}\right) y_{\imath},
\end{aligned}
$$

where $0 \leqq l \leqq \min (k, q)$. If $l=q$, then

$$
D_{q}(k, q ; y)=\left(\begin{array}{c}
n+1 \\
q+1
\end{array}\right) y_{k}-\left(\begin{array}{l}
n \\
q
\end{array}\right) y_{n}-\sum_{\imath=k-q-1}^{k}\left(\begin{array}{c}
i \\
q-k+i+1
\end{array}\right)\left(\begin{array}{c}
n-i-1 \\
k-i
\end{array}\right) y_{\imath} .
$$

By definition,

$$
P_{q}(k, l)=\left(\begin{array}{c}
n+1 \\
q+1
\end{array}\right)-\sum_{j \geq 0}\left(\begin{array}{c}
k+1 \\
l+j+1
\end{array}\right)\left(\begin{array}{c}
n-k \\
q-l-j
\end{array}\right), \quad[5, \text { p. } 182]
$$

where $\left(\begin{array}{l}\alpha \\ \beta\end{array}\right)$ is defined for all integers by the binomial series

$$
(1+x)^{\alpha}=\sum_{\beta=-\infty}^{+\infty}\left(\begin{array}{c}
\alpha \\
\beta
\end{array}\right) x^{\beta}
$$

Proof. We often use the following identities:

$$
\begin{gathered}
\left(\begin{array}{l}
\alpha \\
\beta
\end{array}\right)+\left(\begin{array}{c}
\alpha \\
\beta-1
\end{array}\right)=\left(\begin{array}{c}
\alpha+1 \\
\beta
\end{array}\right) ;\left(\begin{array}{l}
\alpha \\
\beta
\end{array}\right)=0, \quad \text { if } \beta<0 \\
\sum_{\imath+j=\beta}\left(\begin{array}{c}
\alpha+1 \\
i
\end{array}\right)\left(\begin{array}{c}
n-\alpha \\
j
\end{array}\right)=\left(\begin{array}{c}
n+1 \\
\beta
\end{array}\right) \quad[5, \text { p. 194] }
\end{gathered}
$$

and

$$
\sum_{j=q}^{q+r}\left(\begin{array}{c}
p+q+r-j \\
p
\end{array}\right)\left(\begin{array}{l}
j \\
q
\end{array}\right)=\left(\begin{array}{c}
p+q+r+1 \\
r
\end{array}\right) \quad[5, \text { p. 198] }
$$

which directly imply

$$
P_{q}(j-i, l-i)= \begin{cases}0 & \text { if } i \geqq l+1 \\
\left(\begin{array}{l}
n+1 \\
q+1
\end{array}\right) & \text { if } i<l-q\end{cases}
$$

and

$$
P_{q}(k+1, l+1)-P_{q}(k, l)=\left(\begin{array}{c}
k+1 \\
l+1
\end{array}\right)\left(\begin{array}{c}
n-k-1 \\
q-l
\end{array}\right) .
$$

By (25), we can write 
HOLOMORPHIC CURVES

355

(27)

where

$$
D_{q}^{\prime}(k, l ; y)=-\sum_{\jmath=k}^{n-1} \sum_{\imath=-\infty}^{+\infty} P_{q}(j-i, l-i)\left(y_{\jmath-\imath-1}-2 y_{\jmath-i}+y_{\jmath-\imath+1}\right)
$$

and

$$
D_{q}^{\prime \prime}(k, l ; y)=\sum_{j=k}^{n-1} \sum_{\imath=-\infty}^{-1} P_{q}(j-i, l-i)\left(y_{\jmath-\imath-1}-2 y_{\jmath-i}+y_{\jmath-\imath+1}\right) .
$$

Obviously,

$$
\begin{aligned}
& D_{q}^{\prime \prime}(k, l ; y)=\sum_{j=k}^{n-1}\left(P_{q}(j+1, l+1) y_{j}-P_{q}(j, l) y_{\jmath+1}\right) \\
& \quad+\sum_{j=k}^{n-1} \sum_{\imath=j+1}^{+\infty}\left(P_{q}(i+1, l-j+1+i)-2 P_{q}(i, l-j+i)+P_{q}(i-1, l-j+i-1)\right) y_{\imath}
\end{aligned}
$$

Change order summing, we obtain

$$
D_{q}^{\prime \prime}(k, l ; y)=P_{q}(k+1, l+1) y_{k}-P_{q}(n+1, l+1) y_{n}+\sum_{i=k+1}^{n}\left(a_{i}+b_{i}\right) y_{\imath},
$$

where

$$
\begin{aligned}
& a_{\imath}=P_{q}(i+1, l+1)-P_{q}(i-1, l), \\
& b_{i}=\sum_{j=k}^{i-1}\left(P_{q}(i+1, l-j+i+1)-2 P_{q}(i, l-j+i)+P_{q}(i-1, l-j+i-1)\right) .
\end{aligned}
$$

By definition,

$$
\begin{aligned}
& P_{q}(i, l)-P_{q}(i-1, l)=\sum_{j \geq 0}\left\{\left(\begin{array}{c}
i \\
l+j+1
\end{array}\right)\left(\begin{array}{l}
n-i+1 \\
q-l-j
\end{array}\right)-\left(\begin{array}{c}
i+1 \\
l+j+1
\end{array}\right)\left(\begin{array}{c}
n-i \\
q-l-j
\end{array}\right)\right\} \\
& =\sum_{j \geq 0}\left\{\left(\begin{array}{c}
i \\
l+j+1
\end{array}\right)\left[\left(\begin{array}{c}
n-i \\
q-l-j
\end{array}\right)+\left(\begin{array}{c}
n-i \\
q-l-j-1
\end{array}\right)\right]-\left[\left(\begin{array}{c}
i \\
l+j+1
\end{array}\right)+\left(\begin{array}{c}
i \\
l+j
\end{array}\right)\right]\left(\begin{array}{c}
n-i \\
q-l-j
\end{array}\right)\right\} \\
& =\sum_{j \geq 0}\left\{\left(\begin{array}{c}
i \\
l+j+1
\end{array}\right)\left(\begin{array}{c}
n-i \\
q-l-j-1
\end{array}\right)-\left(\begin{array}{c}
i \\
l+j
\end{array}\right)\left(\begin{array}{c}
n-i \\
q-l-j
\end{array}\right)\right\} \\
& =-\left(\begin{array}{c}
i \\
l
\end{array}\right)\left(\begin{array}{l}
n-i \\
q-l
\end{array}\right),
\end{aligned}
$$

which and (26) imply

$$
\begin{aligned}
a_{\imath} & =P_{q}(i+1, l+1)-P_{q}(i, l)+P_{q}(i, l)-P_{q}(i-1, l \\
& =\left(\begin{array}{c}
i+1 \\
l+1
\end{array}\right)\left(\begin{array}{c}
n-i-1 \\
q-l
\end{array}\right)-\left(\begin{array}{l}
i \\
l
\end{array}\right)\left(\begin{array}{c}
n-i \\
q-l
\end{array}\right) \\
& =\left[\left(\begin{array}{c}
i \\
l+1
\end{array}\right)+\left(\begin{array}{l}
i \\
l
\end{array}\right)\right]\left(\begin{array}{c}
n-i-1 \\
q-l
\end{array}\right)-\left(\begin{array}{l}
i \\
l
\end{array}\right)\left(\begin{array}{c}
n-i \\
q-i
\end{array}\right)
\end{aligned}
$$


356

PEICHU NU

$$
=\left(\begin{array}{c}
i \\
l+1
\end{array}\right)\left(\begin{array}{c}
n-i-1 \\
q-l
\end{array}\right)-\left(\begin{array}{l}
i \\
l
\end{array}\right)\left(\begin{array}{l}
n-\imath-1 \\
q-l-1
\end{array}\right) .
$$

By (26), we have

$$
\begin{aligned}
& b_{i}= \sum_{j=k}^{l-1}\left\{\left(\begin{array}{c}
i+1 \\
l-j+i+1
\end{array}\right)\left(\begin{array}{c}
n-i-1 \\
q-l+j-i
\end{array}\right)-\left(\begin{array}{c}
i \\
l-j+i
\end{array}\right)\left(\begin{array}{c}
n-i \\
q-l+j-i+1
\end{array}\right)\right\} \\
&=\sum_{j=k}^{i-1}\left\{\left[\left(\begin{array}{c}
i \\
l-j+i+1
\end{array}\right)+\left(\begin{array}{c}
i \\
l-j+i
\end{array}\right)\right]\left(\begin{array}{c}
n-i-1 \\
q-l+j-i
\end{array}\right)\right.\left.\quad-\left(\begin{array}{c}
i \\
l-j+i
\end{array}\right)\left[\left(\begin{array}{c}
n-i-1 \\
q-l+j-i+1
\end{array}\right)+\left(\begin{array}{c}
n-i-1 \\
q-l+j-i
\end{array}\right)\right]\right\} \\
&=\sum_{j=k}^{2-1}\left\{\left(\begin{array}{c}
i \\
l-j+i+1
\end{array}\right)\left(\begin{array}{c}
n-i-1 \\
q-l+j-i
\end{array}\right)-\left(\begin{array}{c}
i \\
l-j+i
\end{array}\right)\left(\begin{array}{c}
n-i-1 \\
q-l+j-i+1
\end{array}\right)\right\} \\
&=\left(\begin{array}{c}
i \\
l-k+i+1
\end{array}\right)\left(\begin{array}{c}
n-i-1 \\
q-l+k-i
\end{array}\right)-\left(\begin{array}{c}
i \\
l+1
\end{array}\right)\left(\begin{array}{c}
n-i-1 \\
q-l
\end{array}\right) .
\end{aligned}
$$

Hence we finally obtain

$$
\begin{aligned}
& D_{q}^{\prime \prime}(k, l ; y)=P_{q}(k+1, l+1) y_{k}-P_{q}(n+1, l+1) y_{n} \\
& \quad+\sum_{\imath=k+1}^{n}\left\{\left(\begin{array}{c}
i \\
l-k+i+1
\end{array}\right)\left(\begin{array}{c}
n-i-1 \\
q-l+k-i
\end{array}\right)-\left(\begin{array}{l}
i \\
l
\end{array}\right)\left(\begin{array}{c}
n-i-1 \\
q-l-1
\end{array}\right)\right\} y_{\imath} .
\end{aligned}
$$

In similar fashion, we have

(29)

$$
\begin{aligned}
D_{q}^{\prime}(k, l ; y)=-\sum_{j=k}^{n-1} \sum_{\imath=-\infty}^{+\infty}\left(P_{q}(i+1, l-j+i+1)\right. \\
\left.\quad-2 P_{q}(i, l-j+1)+P_{q}(i-1, l-j+i-1)\right) y_{\imath} \\
=-\sum_{\imath=-\infty}^{+\infty} \sum_{j=k}^{n-1}\left\{\left(\begin{array}{c}
i+1 \\
l-j+i+1
\end{array}\right)\left(\begin{array}{c}
n-i-1 \\
q-l+j-i
\end{array}\right)-\left(\begin{array}{c}
i \\
l-j+i
\end{array}\right)\left(\begin{array}{c}
n-i \\
q-l+j-\imath+1
\end{array}\right)\right\} y_{\imath} \\
=-\sum_{\imath=-\infty}^{+\infty} \sum_{j=k}^{n-1}\left\{\left[\begin{array}{c}
i \\
l-j+i+1
\end{array}\right)+\left(\begin{array}{c}
i \\
l-j+i
\end{array}\right)\right]\left(\begin{array}{c}
n-i-1 \\
q-l+j-i
\end{array}\right) \\
\left.\left.-\left(\begin{array}{c}
i \\
l-j+i
\end{array}\right)\left[\begin{array}{c}
n-i-1 \\
q-l+j-i+1
\end{array}\right)+\left(\begin{array}{c}
n-i-1 \\
q-l+j-i
\end{array}\right)\right]\right\} y_{\imath} \\
=-\sum_{i=-\infty}^{+\infty} \sum_{==k}^{n-1}\left\{\left(\begin{array}{c}
i \\
l-j+i+1
\end{array}\right)\left(\begin{array}{c}
n-i-1 \\
q-l+j-i
\end{array}\right)-\left(\begin{array}{c}
i \\
l-j+i
\end{array}\right)\left(\begin{array}{c}
n-i-1 \\
q-l+j-i+1
\end{array}\right)\right\} y_{\imath} \\
=-\sum_{i=-\infty}^{+\infty}\left\{\left(\begin{array}{c}
i \\
l-k+i+1
\end{array}\right)\left(\begin{array}{c}
n-i-1 \\
q-l+k-i
\end{array}\right)-\left(\begin{array}{c}
i \\
l-n+i+1
\end{array}\right)\left(\begin{array}{c}
n-i-1 \\
q-l+n-i
\end{array}\right)\right\} y_{i} \\
=-\sum_{i=k-l-1}^{n}\left(\begin{array}{c}
i \\
l-k+i+1
\end{array}\right)\left(\begin{array}{c}
n-i-1 \\
q-l+k-i
\end{array}\right) y_{i}+\left(\begin{array}{c}
n \\
l+1
\end{array}\right)\left(\begin{array}{c}
-1 \\
q-l
\end{array}\right) y_{n},
\end{aligned}
$$


because

$$
\begin{aligned}
& y_{\imath}=0 \quad \text { if } i>n, \\
& \left(\begin{array}{c}
i \\
l-k+i+1
\end{array}\right)=0 \quad \text { if } i<k-l-1, \\
& \left(\begin{array}{c}
n-i-1 \\
q-l+n-i
\end{array}\right)=0 \quad \text { if } i \leqq n-1 .
\end{aligned}
$$

Thus (27), (28) and (29) imply (22). q.e.d.

COROLLARY.

$$
C_{k}^{q} \equiv \sum_{j=k}^{n-1} \sum_{i=0}^{q} P_{q}(j-i, q-i)=\frac{n-k}{2}\left\{(n+1)\left(\begin{array}{c}
n \\
q
\end{array}\right)-(k-q)\left(\begin{array}{c}
n+1 \\
q
\end{array}\right)\right\} .
$$

Proof. In Lemma 3 we let

$$
y_{s}= \begin{cases}j & \text { if } j \leqq n \\ 0 & \text { if } j>n,\end{cases}
$$

then $D_{q}(k, q ; y)=0$, so we have

$$
\sum_{i=k-1}^{k}\left(\begin{array}{c}
i \\
q-k+i+1
\end{array}\right)\left(\begin{array}{c}
n-i-1 \\
k-i
\end{array}\right) i=\left(\begin{array}{c}
n+1 \\
q+1
\end{array}\right) k-\left(\begin{array}{l}
n \\
q
\end{array}\right) n
$$

In Lemma 3 we take

$$
y_{j}= \begin{cases}j(j+1) & \text { if } j \leqq n \\ 0 & \text { if } j>n .\end{cases}
$$

Then

$$
C_{k}^{q}=-\frac{1}{2} D_{q}(k, q ; y)=\frac{n(n+1)}{2}\left(\begin{array}{l}
n \\
q
\end{array}\right)-\frac{k(k+1)}{2}\left(\begin{array}{l}
n+1 \\
q+1
\end{array}\right)+I,
$$

where

$$
\begin{aligned}
I & =\frac{1}{2} \sum_{i=k-q-1}^{k}\left(\begin{array}{c}
i \\
q-k+i+1
\end{array}\right)\left(\begin{array}{c}
n-i-1 \\
k-i
\end{array}\right) i(i+1) \\
& =\frac{k-q}{2} \sum_{2=k-q-1}^{k}\left(\begin{array}{c}
i+1 \\
q-k+i+1
\end{array}\right)\left(\begin{array}{c}
n-i-1 \\
k-i
\end{array}\right) i \\
& =\frac{k-q}{2}\left\{\sum_{2=k-q-1}^{k}\left(\begin{array}{c}
i \\
q-k+i+1
\end{array}\right)\left(\begin{array}{c}
n-i-1 \\
k-i
\end{array}\right) i+\sum_{2=k-q}^{k}\left(\begin{array}{c}
i \\
q-k+i
\end{array}\right)\left(\begin{array}{c}
n-i-1 \\
k-i
\end{array}\right) i\right\} .
\end{aligned}
$$

By (31), we have

$$
I=\frac{k-q}{2}\left\{k\left(\begin{array}{c}
n+1 \\
q+1
\end{array}\right)-n\left(\begin{array}{l}
n \\
q
\end{array}\right)+k\left(\begin{array}{c}
n+1 \\
q
\end{array}\right)-n\left(\begin{array}{c}
n \\
q-1
\end{array}\right)\right\} .
$$

Thus (33) and (32) imply (30). q.e.d. 


\section{Proof of Theorem 2 .}

We have the following inequalities [5]

$$
\begin{aligned}
\sum_{A^{q}} m_{k}\left(A^{q}\right)= & -\sum_{\jmath=k}^{n-1} \sum_{\imath=0}^{q} P_{q}(j-i, q-i)\left(E+S_{\jmath-i}+T_{\jmath-\imath-1}\right. \\
& \left.-2 T_{\jmath-i}+T_{\jmath-\imath+1}\right)+\mu\left(T^{2}\right), \quad \text { if } 0 \leqq q \leqq k \quad[5, \text { p. 193] }
\end{aligned}
$$

and

$$
\begin{aligned}
\sum_{A^{q}} m_{k}\left(A^{q}\right)= & -\sum_{j=0}^{k} \sum_{\imath=0}^{n-q-1} P_{n-q-1}(n-j-i-1, n-q-i-1)\left(E+S_{j+i}\right. \\
& \left.+T_{j+\imath-1}-2 T_{\jmath+i}+T_{j+\imath+1}\right)+\mu\left(T^{2}\right), \quad \text { if } q \geqq k . \quad[5, \text { p. 201] }
\end{aligned}
$$
have

Firstly, let us deduce (4) for the case $0 \leqq q \leqq k$. By (1) and Lemma 3, we

$$
\begin{gathered}
\sum_{A^{q}} m_{k}\left(A^{q}\right)=D_{q}\left(k, q ; T^{0}\right)+C_{k}^{q} H+\mu\left(T^{2}\right) \\
=\left(\begin{array}{c}
n+1 \\
q+1
\end{array}\right) T_{k}^{0}-\left(\begin{array}{c}
n \\
q
\end{array}\right) N_{n}+\sum_{\imath=k-q-1}^{k}\left(\begin{array}{c}
i \\
q-k+i+1
\end{array}\right)\left(\begin{array}{c}
n-i-1 \\
k-i
\end{array}\right)\left(-T_{\imath}^{0}\right) \\
+C_{k}^{q} H+\mu\left(T^{2}\right) .
\end{gathered}
$$

By Corollary 1 of Lemma 2 and (24), the sum of the right hand side of the above identity equals

$$
\begin{aligned}
& -\sum_{\imath=k-1}^{k} \frac{i+1}{k+1}\left(\begin{array}{c}
i \\
q-k+i+1
\end{array}\right)\left(\begin{array}{c}
n-i-1 \\
k-i
\end{array}\right) T_{k}^{0} \\
& +\frac{1}{2} \sum_{\imath=k-q-1}^{k}\left(\begin{array}{c}
i \\
q-k+i+1
\end{array}\right)\left(\begin{array}{c}
n-i-1 \\
k-i
\end{array}\right)(k-i)(i+1) H+\mu(T) \\
& =-\frac{k-q}{k+1}\left(\begin{array}{c}
n+1 \\
q+1
\end{array}\right) T_{k}^{0}+\frac{1}{2}(n-k)(k-q)\left(\begin{array}{c}
n+1 \\
q
\end{array}\right) H+\mu(T),
\end{aligned}
$$

where

$$
\begin{aligned}
& \sum_{i=k-q-1}^{k} \frac{i+1}{k+1}\left(\begin{array}{c}
i \\
q-k+i+1
\end{array}\right)\left(\begin{array}{c}
n-i-1 \\
k-i
\end{array}\right) \\
= & \frac{k-q}{k+1} \sum_{\imath=k-q-1}^{k}\left(\begin{array}{c}
i+1 \\
q-k+i+1
\end{array}\right)\left(\begin{array}{c}
n-i-1 \\
k-i
\end{array}\right) \\
= & \frac{k-q}{k+1} \sum_{\jmath=k-q}^{k+1}\left(\begin{array}{c}
n-j \\
n-k-1
\end{array}\right)\left(\begin{array}{c}
j \\
k-q
\end{array}\right)=\frac{k-q}{k+1}\left(\begin{array}{c}
n+1 \\
q+1
\end{array}\right) \quad \text { (by (24)) }
\end{aligned}
$$

and 


$$
\begin{aligned}
& \sum_{i=k-1}^{k}\left(\begin{array}{c}
i \\
q-k+i+1
\end{array}\right)\left(\begin{array}{c}
n-i-1 \\
k-i
\end{array}\right)(k-\imath)(i+1) \\
= & (n-k)(k-q) \sum_{i=k-q-1}^{k}\left(\begin{array}{c}
i+1 \\
q-k+i+1
\end{array}\right)\left(\begin{array}{c}
n-i-1 \\
k-i-1
\end{array}\right) \\
= & (n-k)(k-q) \sum_{j=k-q}^{k+1}\left(\begin{array}{c}
n-j \\
n-k
\end{array}\right)\left(\begin{array}{c}
j \\
k-q
\end{array}\right) \\
= & (n-k)(k-q)\left\{\left(\begin{array}{c}
n+1 \\
q
\end{array}\right)+\left(\begin{array}{c}
k+1 \\
k-q
\end{array}\right)\left(\begin{array}{c}
n-k-1 \\
n-k
\end{array}\right)\right\} \quad \text { (by (24)) } \\
= & (n-k)(k-q)\left(\begin{array}{c}
n+1 \\
q
\end{array}\right) .
\end{aligned}
$$

Hence (30), (36) and Lemma 1 imply

$$
\sum_{A^{q}} m_{k}\left(A^{q}\right)=\left(\begin{array}{c}
n \\
q
\end{array}\right)\left(\frac{n+1}{k+1} T_{k}^{0}-N_{n}+\frac{1}{2}(n+1)(n-k) H\right)+\mu\left(T^{2}\right) .
$$

Next, we deduce (4) for the case $q \geqq k$. Take $y_{\jmath}=T_{n-1-\jmath}^{0}$ in Lemma 3 . Then (23) and (35) imply

$$
\begin{aligned}
& \sum_{A^{q}} m_{k}\left(A^{q}\right)=D_{n-q-1}(n-k-1, n-q-1 ; y)+C_{n-k-1}^{n-q-1} H+\mu\left(T^{2}\right) \\
& =\left(\begin{array}{c}
n+1 \\
q+1
\end{array}\right) T_{k}^{0}-\sum_{j=k}^{n-q+k}\left(\begin{array}{c}
n-1-j \\
q-k-1
\end{array}\right)\left(\begin{array}{c}
j \\
k
\end{array}\right) T_{j}^{0}+C_{n-k-1}^{n-q-1} H+\mu\left(T^{2}\right),
\end{aligned}
$$

$\left(y_{n}=T_{-1}^{0}=0\right)$. By Corollary 1 of Lemma 2 and (24), the sum of the right hand side of the above equals

$$
\begin{aligned}
& -\sum_{j=k}^{n-q+k} \frac{n-j}{n-k}\left(\begin{array}{c}
n-1-j \\
q-k-1
\end{array}\right)\left(\begin{array}{l}
j \\
k
\end{array}\right) T_{k}^{0}+\frac{1}{2} \sum_{j=k}^{n-q+k}(j-k)(n-j)\left(\begin{array}{c}
n-1-j \\
q-k-1
\end{array}\right)\left(\begin{array}{l}
j \\
k
\end{array}\right) H \\
& -\sum_{j=k}^{n-q+k} \frac{j-k}{n-k}\left(\begin{array}{c}
n-1-j \\
q-k-1
\end{array}\right)\left(\begin{array}{l}
j \\
k
\end{array}\right) N_{n}+\mu(T) \\
= & -\frac{q-k}{n-k}\left(\begin{array}{c}
n+1 \\
q+1
\end{array}\right) T_{k}^{0}+\frac{1}{2}(k+1)(q-k)\left(\begin{array}{c}
n+1 \\
q+2
\end{array}\right) H-\frac{k+1}{n-k}\left(\begin{array}{c}
n \\
q+1
\end{array}\right) N_{n}+\mu(T),
\end{aligned}
$$

where

$$
\begin{aligned}
& \sum_{j=k}^{n-q+k} \frac{n-j}{n-k}\left(\begin{array}{c}
n-1-j \\
q-k-1
\end{array}\right)\left(\begin{array}{l}
j \\
k
\end{array}\right) \\
= & \frac{q-k}{n-k} \sum_{j=k}^{n-q+k}\left(\begin{array}{l}
n-j \\
q-k
\end{array}\right)\left(\begin{array}{l}
j \\
k
\end{array}\right)=\frac{q-k}{n-k}\left(\begin{array}{l}
n+1 \\
q+1
\end{array}\right), \quad(\text { by }(24))
\end{aligned}
$$




$$
\begin{aligned}
& \sum_{j=k}^{n-q+k}(j-k)(n-j)\left(\begin{array}{l}
n-1-j \\
q-k-1
\end{array}\right)\left(\begin{array}{l}
j \\
k
\end{array}\right) \\
= & (k+1)(q-k) \sum_{j=k}^{n-q+k}\left(\begin{array}{l}
n-j \\
q-k
\end{array}\right)\left(\begin{array}{c}
j \\
k+1
\end{array}\right) \\
= & (k+1)(q-k)\left(\begin{array}{c}
n+1 \\
q+2
\end{array}\right) \quad(\text { by (24)) }
\end{aligned}
$$

and

$$
\begin{aligned}
& \sum_{j=k}^{n-q+k} \frac{j-k}{n-k}\left(\begin{array}{c}
n-1-j \\
q-k-1
\end{array}\right)\left(\begin{array}{l}
j \\
k
\end{array}\right) \\
= & \frac{k+1}{n-k} \sum_{\jmath=k+1}^{n-q+k}\left(\begin{array}{c}
n-1-j \\
q-k-1
\end{array}\right)\left(\begin{array}{c}
j \\
k+1
\end{array}\right) \\
= & \frac{k+1}{n-k}\left(\begin{array}{c}
n \\
n-q-1
\end{array}\right)=\frac{k+1}{n-k}\left(\begin{array}{c}
n \\
q+1
\end{array}\right), \quad \text { (by (24)) }
\end{aligned}
$$

Hence (30), (38) and Lemma 1 imply

$$
\sum_{A^{q}} m_{k}\left(A^{q}\right)=\frac{n-q}{n-k}\left(\begin{array}{c}
n+1 \\
q+1
\end{array}\right) T_{k}^{0}-\frac{k+1}{n-k}\left(\begin{array}{c}
n \\
q+1
\end{array}\right) N_{n}+\frac{(k+1)(n+1)}{2}\left(\begin{array}{c}
n \\
q+1
\end{array}\right) H+\mu\left(T^{2}\right) .
$$

Now (4) follows from (37) and (39).

Finally, by (3), we have

$$
\frac{n+1}{k+1} N_{k}(r)-N_{n}(r)+\frac{(n+1)(n-k)}{2} H(r)=-Q_{k}(r)-\frac{(n+1)(n-k)}{2} E(r)
$$

which implies (5). $\quad$ q.e.d.

\section{Discussion.}

In this section, we assume that $V$ has an infinite harmonic exhaustion and the conditions in Theorem 2 hold. Define

$$
\theta_{k}=\lim _{r \rightarrow \infty} \sup \frac{Q_{k}(r)}{T_{k}(r)} \text { and } \Theta_{k}=\lim _{r \rightarrow \infty} \sup \frac{N_{n}(r)}{T_{k}^{0}(r)} .
$$

For each $q$-dimensional projective subspace $A^{q}$ of $\boldsymbol{P}_{n}$, we define the defects of $A^{q}$ to be:

$$
\delta_{k}\left(A^{q}\right)=\lim _{r \rightarrow \infty} \inf \frac{m_{k}\left(r, A^{q}\right)}{T_{k}(r)} \quad \text { and } \quad \Delta_{k}\left(A^{q}\right)=\lim _{r \rightarrow \infty} \inf \frac{m_{k}\left(r, A^{q}\right)}{T_{k}^{0}(r)} .
$$

Clearly $0 \leqq \Delta_{k}\left(A^{q}\right) \leqq \delta_{k}\left(A^{q}\right) \leqq 1$. If $H_{k}<+\infty$, by Theorem 2, (20) and Corollary 2 of Lemma 2 we have

$$
\sum_{A^{q}} \Delta_{k}\left(A^{q}\right) \leqq \varepsilon_{k}^{q}\left(\begin{array}{l}
n \\
q
\end{array}\right)\left\{\frac{n+1}{k+1}-\Theta_{k}+\frac{(n+1)(n-k)}{2} H_{k}\right\} .
$$


If $\chi_{k}<+\infty$, by Theorem 2, (20) and (21) we have

$$
\sum_{A^{q}} \delta_{k}\left(A^{q}\right) \leqq \varepsilon_{k}^{q}\left(\begin{array}{l}
n \\
q
\end{array}\right)\left\{\frac{n+1}{k+1}-\theta_{k}+\frac{(n+1)(n-k)}{2} \chi_{k}\right\} .
$$

Let $Z(\tilde{x})$ be the set of zero points of $|\tilde{x}|$. Let $\nu_{p}(f)$ denote the order of zero at $p$ of a function $f$ on $V$. Clearly, we see

Define

$$
\nu_{p}\left(\left|X_{\gamma}^{1}\right|\right) \geqq 2 \nu_{p}(|\tilde{x}|)-1, \quad \text { if } p \in Z(\tilde{x}) .
$$

$$
s_{p}=\left\{\begin{array}{l}
\text { the stationary index of } x \text { at } p, \quad \text { if } p \text { is a critical point of } x \\
0 \quad \text { otherwise }
\end{array}\right.
$$

and

$$
I_{p}=\nu_{p}\left(\left|X_{\gamma}^{1}\right|\right)-2 \nu_{p}(|\tilde{x}|)-s_{p} .
$$

We can prove that

$$
I_{p}=0, \quad \text { if } p \in V-Z(\tilde{x}) .
$$

Define

$$
i(t)=\sum_{p \in V[t]} I_{p} \quad \text { and } \quad I(r)=\int_{r_{0}}^{r} i(t) d t
$$

Then

$$
I(r)=N_{1}(r)-2 N_{0}(r)-S_{0}(r) .
$$

Hence (1) implies

$$
E(r)+H(r)=I(r) .
$$

If $V=C$ or $C-\{0\}$, we can choose $\tilde{x}$ such that $Z(\tilde{x})=\varnothing$, so

$$
H(r)=-E(r) .
$$

If $x$ is transcendental and $Z(\tilde{x})$ is a finite set, then

$$
\lim _{r \rightarrow \infty} \frac{I(r)}{T_{0}(r)}=0 \text {. }
$$

\section{REFERENCES}

[1] Ahlfors, L., The theory of meromorphic curves, Acta Soc. Sci. Fenn. Nova Ser. A3 (4) (1941), 171-183.

[2] Cartan, H., Sur les zéros des combinaisons linéaires de $p$ fonctions holomorphes données, Mathematica (Cluj) 7 (1933), 5-31.

[3] Nevanlinna, R., Le théorème de Picard-Borel et la théorie des fonctions meromorphes, Gauthiers-Villars, Paris, 1929.

[4] Shiffman, B., Holomorphic curves in algebraic manifolds, Bull. Amer. Math. 
Soc. 83 (1977), 553-568.

[5] Wu, H., The equidistribution theory of holomorphic curves, Annals of Math. Studies 64, Princeton Univ. Press, Princeton, NJ. (1970).

Department of Mathematics

SHANDONG UNIVERSITY

Jinan, Shandong, China 